\title{
Inscribing a symmetric body in an ellipse.
}

\author{
Francisco Santos* \\ Dept. de Matemáticas, Estadística y Computación. \\ Universidad de Cantabria. \\ e_mail: santos@matsun1.unican .es
}

July 1,1996

\begin{abstract}
We prove that any bounded, centrally symmetric object $K$ in the plane can be inscribed in an ellipse $E$ touching its boundary $\partial K$ at at least four points. An application to Minkowski geometry is given.

Keywords : Computational Geometry, Convexity
\end{abstract}

\section{Introduction}

There is a wide range of problems related to inscribing different kinds of (usually convex) bodies into one another. For example, it is well-known that one can always inscribe or circumscribe a square around a convex body in the plane. Also, S. Kakutani [8] has shown that one can always circumsbribe a cube around a bounded closed convex set in any finite dimension. On the negative side, Eggleton [6] has constructed convex bodies in the plane in which an $n$-gon, $n>4$ cannot be inscribed and convex bodies in $\mathbf{R}^{3}$ in which a cube cannot be inscribed, or a regular octahedron circumscribed. We finally mention the result by F. Behrend [2] (for dimension 2) and Zaguskin [10] (for arbitrary dimension), that there is an ellipsoid with minimal (resp. maximal) volume circumscribed (resp. inscribed) on any convex body.

This note is devoted to prove the following result, illustrated in Figure 1.

Theorem 1 Let $K$ be a bounded, centrally symmetric body in the Euclidean plane $\mathbf{R}^{2}$, not contained in a straight line. Then, there exists an ellipse $E$ containing $K$ and such that the boundaries $\partial K$ and $\partial E$ intersect in at least two pairs of opposite points.

\footnotetext{
*Partially supported by DGICyT PB 92/0498-C02.
} 


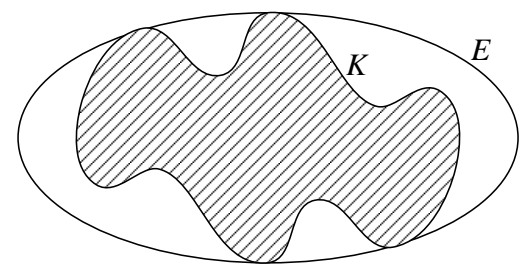

Figure 1.

For convenience, in Theorem 1 and in the rest of the paper the word ellipse (also the word circle) will be used meaning not only the curve but also its interior region. We will say boundary of an ellipse/circle (or use the symbol $\partial$ ) when referring to the curve.

Theorem 1 is not intuitively surprising at all. An easy (but not valid) heuristics for finding the circumscribed ellipse $E$ goes as follows: let $O$ be the center of symmetry of $K$ and let $C$ be the smallest circle containing $K$ with center at $O$. The boundaries of $C$ and $K$ intersect in a pair of opposite points $p$ and $q^{\prime}$. Then one normally can shrink $C$ in the direction perpendicular to the segment $\left[x, x^{\prime}\right]$ until a second pair of opposite points of intersection appears.

The mistake in the argument above is that even the slightest shrinking of the enclosing circle may force a small part of the circle coming into the interior of $K$. This will happen whenever the circle and the body $K$ have the same curvature radius at $p$ and $q$. For example, let $K$ be defined by the equation $\left(x^{2}+2 y^{2}+2 y\right)\left(x^{2}+2 y^{2}-2 y\right) \leq 0$ and $C$ the unit circle, as in Figure 2.

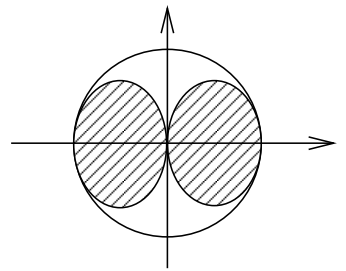

Figure 2.

Section 2 contains the proof of Theorem 1. Section 3 shows an application of the result in the context of Minkowskian geometry. The natural generalization of Theorem 1 to higher dimension would be that an ellipsoid could be circumscribed around any convex body in $\mathbf{R}^{n}$ through $2 n$ points. We do not know a proof of this.

\section{Proof of the theorem}

Lemma 2 Let $K$ be a compact, centrally symmetric, convex body in the plane $\mathbf{R}^{2}$, not contained in any straight line. Then, there exists an ellipse $E$ such that $\partial K$ and $\partial E$ intersect in at least three pairs of opposite points. 
Proof: Let $P, P^{\prime}, Q$ and $Q^{\prime}$ be any two pairs of opposite points in $\partial K$, not in a straight line. Then, either there exists a third pair of opposite points $R, R^{\prime}$ in $\partial K$ such that $P Q R P^{\prime} Q^{\prime} R^{\prime}$ is a strictly convex, centrally symmetric hexagon, or $K$ coincides with the cuadrilateral $P Q P^{\prime} Q^{\prime}$ (recall that $K$ is assumed to be convex). In the first case there exists an ellipse passing through the six points. In the second case, if we slightly reduce any ellipse passing through $P, Q, P^{\prime}$ and $Q^{\prime}$ we will obtain an ellipse passing through four pairs of opposite points of the boundary of the cuadrilateral.

Lemma 3 Let $K$ be a compact, centrally symmetric, convex body in the plane $\mathbf{R}^{2}$, not contained in any straight line. Then, there exists a circle $C$ and a linear transformation l of the plane such that the image $M$ of $K$ through $l$ is contained in $C$ and the boundaries $\partial M$ and $\partial C$ intersect in at least two pairs of opposite points.

Proof: Let us apply Lemma 2 to $K$ and then make a linear transformation $l^{\prime}$ in the plane sending the ellipse $E$ obtained there into a circle $C^{\prime}$. Let $M^{\prime}=l^{\prime}(K)$. Let $f:[0,2 \pi] \rightarrow \mathbf{R}_{+}$be the map describing $\partial M^{\prime}$ in polar coordinates, as a function of the angle. Then, $f$ is periodical of period $\pi$ (because $M^{\prime}$ is centrally symmetric) and takes the same value in three different points $0 \leq x<y<z<\pi$ (the points where $\partial M^{\prime}$ intersects the circle $C^{\prime}$ ). In these conditions, $f$ has at least two local maxima in a period. In fact, either at least two of the open intervals $(x, y),(y, z)$ and $(z, x+\pi)$ contain a local maximum of $f$, or one of them (say $(x, y)$ ) contains a local maximum and the third point (i.e. $z$ ) is another local maximum, or the three points $x, y$ and $z$ are local maxima.

Let $\alpha$ and $\beta$ be two local maxima of $f$ in the period $[0, \pi)$ and suppose without loss of generality that $\alpha$ is actually a global maximum. Consider the collection of linear transformations $l_{r}(0<r \leq 1)$ that fix the direction of $\beta$ and that contract its perpendicullar direction by a ratio $r$. Call $f_{r}$ the transformed of $f$ by $l_{r}$, i.e. $f_{r}=f \circ l_{r}$. Then, for $r$ close to $0 \beta$ is clearly a global maximum of $f_{r}$. Call $r_{0}$ the supremum of the values of $r$ for which this happens. Our claim is that in these conditions $\beta$ is a global maximum for $f_{r_{0}}$, but it is not the only one.

To prove the claim, the fact that $\beta$ is a global maximum of $f_{r}$, for $r$ arbitrarily close to $r_{0}$ implies that it is also a global maximum of $f_{r_{0}}$. On the other hand, for any $r>r_{0}$ the absolute maximum of $f_{r}$ is not attained on $\beta$, nor in a certain interval $[\beta-\epsilon, \beta+\epsilon]$ around $\beta$ (because $\beta$ is a local maximum of every $f_{r}$ ). Consider a sequence $r_{1}>r_{2}>\ldots$ with limit $r_{0}$, and for every $r_{i}$ let $\gamma_{i}$ be an absolute maximum of $l_{r_{i}}$. Then the sequence $\gamma_{i}$ has at least one limit point $\gamma$ in the compact $[0, \beta-\epsilon] \cup[\beta+\epsilon, \pi]$ and this limit point must be an absolute maximum of $f_{r_{0}}$.

The claim finishes the proof of the lemma as follows. Let $l=l_{r_{0}} \circ l^{\prime}, M=$ $l(K)=l_{r_{0}}\left(M^{\prime}\right)$ and $C$ be the circle of radius $f_{r_{0}}(\beta)$. This circle contains $M$ and the boundaries $\partial M$ and $\partial C$ intersect in the two pairs of opposite points in the directions of $\beta$ and $\gamma$. 
Proof: (of Theorem 1) If $K$ is convex let us apply lemma 3 to it, and obtain a circle $C$ and a linear transformation $l$ sending $K$ to a convex $M$ in such a way that $M \subseteq C$ and $\partial C \cap \partial M$ consists on at least two pairs of opposite points. The inverse image $E=l^{-1}(C)$ is an ellipse in the conditions of Theorem 1 .

If $K$ is not convex, apply the previous remark to its convex hull conv $(K)$. We will prove that any point in $\partial \operatorname{conv}(K) \cap \partial E$ is also in $\partial K \cap \partial E$, and that will finish the proof. Let $P$ be one of the intersection points in $\partial \operatorname{conv}(K) \cap \partial E$.

As we have $\partial \operatorname{conv}(K) \subset \operatorname{conv}(K)=\operatorname{conv}(\partial K), P$ is contained in a segment $[Q, R]$ with $Q, R \in \partial K \subseteq \bar{E}$. Then, as $P \in \partial E$, the only possibility is $P=Q$ or $P=R$. Thus, $P \in \partial K$.

\section{An application to Minkowski geometry.}

A bounded convex body $K$ in $\mathbf{R}^{n}$, centrally symmetric respect to the origin $O$ defines a Minkowski distance funtction (to be called the $K$-distance) as follows. The $K$-distance between a point $P$ and a point $Q$ equals the unique scaling factor $\lambda$ for which $Q$ lies in the boundary of $P+\lambda Q$. These distance functions include all the $\mathrm{L}^{p}$ metrics and, actually, all the metrics compatible with a norm of the vector space $\mathbf{R}^{n}$.

Chew and Drysdale [3] used the name convex distance functions for the Minkowski distances and showed a divide-and-conquer algorithm for computing Voronoi diagrams with respect to them. Such Voronoi diagrams have further been studied in [4], [5] and [7] (the later in 3-space).

$K$ is the unit ball of the $K$-distance, and its boundary $\partial K$ the unit circle. All the other $K$-circles are scaled translations of $\partial K$. If the convex $K$ is strictly convex and smooth, then the $K$-circles satisfy the following good properties:

(i) there is a unique $K$-circle passing through any given three non-collinear points, and there is no $K$-circle passing through three collinear points.

(ii) if $A, B, C$ and $D$ are the four vertices of a convex cuadrilateral in consecutive order, and no $K$-circle passes through the four points, then either $A$ and $B$ lie outside and $C$ and $D$ lie inside the $K$-circles passing through the other three points, or viceversa.

In [5] Theorem 1 is used (without proof) to show that the topological types of Delaunay triangulations which can appear with a metric whose ball is not elliptical differ from those obtained for elliptical balls (the latter are the same as those of the Euclidean distance). In the same context, we are going to apply Theorem 1 to show that a certain theorem of Euclidean geometry translates to "Minkowskian" geometry if and only if the unit ball $K$ is an ellipse. The construction in the proof of the following theorem is based in [9]. Asplund and Grümbaum [1] have also given a characterization of ellipses in terms of a property of triangles of the Minkowskian geometry. 
Theorem 4 Let $K$ be a bounded, smooth, strictly convex and symmetric body in the plane. Then, $K$ is an ellipse if and only if the following statement is satisfied for the metric associated with $K$ :

Let $p_{1}, \ldots, p_{8}$ be eight distinct points in the plane. If the quadruples of points $\left.\left[p_{1}, p_{2}, p_{5}, p_{6}\right], p_{3}, p_{4}, p_{7}, p_{8}\right],\left[p_{1}, p_{2}, p_{3}, p_{4}\right],\left[p_{3}, p_{4}, p_{5}, p_{6}\right]$ and $\left[p_{5}, p_{6}, p_{7}, p_{8}\right]$ are all collinear or $K$-cocircular, then $\left[p_{1}, p_{2}, p_{7}, p_{8}\right]$ is also collinear or $K$-cocircular.

Proof: The statement is a well-known and easy to proof theorem of Euclidean geometry, and will still be valid for convex distance functions with elliptical balls, by an affine transformation argument. We will consider a non-elliptical, strictly convex, smooth and symmetric body $K$ and construct a counterexample to the statement.

Let us apply Theorem 1 to $K$ and, without loss of generality, suppose that the ellipse $E$ obtained is actually an Euclidean circle (we can make a linear transformation to $K$, if needed). Let $A, B, C$ and $D$ be two pairs of opposite points in $\partial K \cap \partial E$. Let $A^{\prime}, B^{\prime}, C^{\prime}$ and $D^{\prime}$ be other four points in $\partial E$, with the segments $\left[A, A^{\prime}\right],\left[B, B^{\prime}\right],\left[C, C^{\prime}\right]$ and $\left[D, D^{\prime}\right]$ being of equal (small) length (see Figure 3 (a) ). Since $K$ does not coincide with $E$, we can assume that at least $A^{\prime}$ and $C^{\prime}$ lie outside $K$.

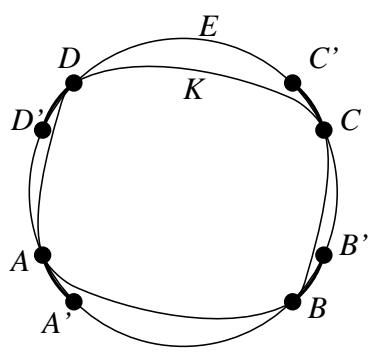

(a)

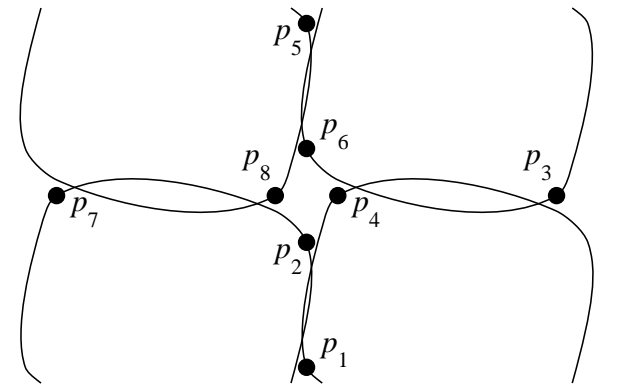

(b)

Figure 3 .

Let $S$ consist on the eight points in Figure 3 (b), obtained as

$$
\begin{aligned}
& p_{1}=A, \quad p_{2}=D^{\prime}, \quad p_{3}=C^{\prime}, \quad p_{4}=D, \\
& p_{5}=p_{4}+\left(D^{\prime}-A^{\prime}\right), \quad p_{6}=p_{4}+\left(A-A^{\prime}\right), \\
& p_{7}=p_{6}+\left(A^{\prime}-B^{\prime}\right)=p_{2}+(D-C), \quad p_{8}=p_{6}+\left(B-B^{\prime}\right)=p_{2}+\left(C^{\prime}-C\right) \text {. }
\end{aligned}
$$

By construction, the quadruples $\left[p_{1}, p_{2}, p_{5}, p_{6}\right]$ and $\left[p_{3}, p_{4}, p_{7}, p_{8}\right]$ are collinear. In the other hand, the points $p_{1}, p_{3}, p_{5}$ and $p_{7}$ lie (respectively) inside the $K$-circles passing through $\left[p_{2}, p_{3}, p_{4}\right],\left[p_{4}, p_{5}, p_{6}\right],\left[p_{6}, p_{7}, p_{8}\right]$ and $\left[p_{8}, p_{1}, p_{2}\right]$. 
We can one-by-one move the points $p_{5}, p_{3}$ and $p_{1}$ along the lines $p_{1} p_{2} p_{5} p_{6}$ and $p_{3} p_{4} p_{7} p_{8}$ to a position where the quadruples $\left[p_{5}, p_{6}, p_{7}, p_{8}\right],\left[p_{3}, p_{4}, p_{5}, p_{6}\right]$ and $\left[p_{1}, p_{2}, p_{3}, p_{4}\right]$ are $K$-cocircular (the movements will be towards the exterior of the figure). After these movements $p_{7}$ will still be outside the $K$-circle passing through $p_{1}, p_{2}$ and $p_{8}$.

\section{References}

[1] E. Asplund and B Grümbaun, On the geometry of Minkowski planes, L'Enseignement Math. 6 (1960), 299-306.

[2] F. Behrend, Über die Kleinste umbeschriebene und die grösste einbeschriebene Ellipse eines konvexen Bereichs, Math. Ann. 115 (1938), 379411.

[3] L.P. Chew and R.L. Drysdale III, Voronoi Diagrams Based on Convex Distance Functions, in Proceedings 1st ACM Symposium on Computational Geometry, 235-244, ACM Press, 1985.

[4] A.G. Corbalán, M. Mazón and T. Recio, Geometry of bisectors for strictly convex distances, Journal of Computational Geometry and its applications, in press.

[5] A.G. Corbalán, M. Mazón, T. Recio and F. Santos, On the Topological Shape of Planar Voronoi Diagrams, in Proceedings 9th ACM Symposium on Computational Geometry, 109-115, ACM Press, 1993.

[6] H.G. Eggleton, Figures inscribed in convex sets, Amer. Math. Monthly 65 (1958) 76-80.

[7] C. Icking, R. Klein, N.M. Le and L. Ma, Convex Distance Functions in 3space are Different, Fundamenta Informaticae 22 (1995) 331-352. Extended abstract in Proceedings 9th ACM Symposium on Computational Geometry, 116-123, ACM Press, 1993.

[8] Shizuo Kakutani, A proof that there exists a circumscribing cube around any bounded closed convex set in $\mathbf{R}^{3}$, Annal. of Math. 43 (1942), 739-741.

[9] F. Santos, On Delaunay oriented matroids for convex distance functions, Discrete and Computational Geometry, to appear. Extended abstract in Proceedings of the 6th Canadian Conference on Computational Geometry, University of Saskatoon, August 1994.

[10] V.L. Zaguskin, On circumscribed and inscribed ellipsoids of extremal volume, Uspehi Mat. Nauk 13 (1958), 89-92. (Russian). English translation in Russian Mathematical surveys. 